\title{
Effect of the Laser Heating of Nanotube Nuclei on the Nanotube Type Population
}

\author{
Pavel Nikolaev ${ }^{1}(\bowtie)$, William Holmes ${ }^{2}$, Edward Sosa ${ }^{3}$, Peter Boul ${ }^{3}$, and Sivaram Arepalli ${ }^{1}$ \\ ${ }^{1}$ Department of Energy Science, Sungkyunkwan University, 300 Cheoncheon-dong, Jangan-gu, Suwon 440-746, Korea \\ ${ }^{2}$ Alan G. MacDiarmid NanoTech Institute, The University of Texas at Dallas, 800 West Campbell Road, Richardson, TX 75080, \\ USA \\ ${ }^{3}$ ERC Inc. / NASA Johnson Space Center, Mail Stop ES-4, P.O.Box 58561, Houston, TX 77258, USA \\ Received: 16 April 2009 / Revised: 31 August 2009 / Accepted: 1 September 2009 \\ CTsinghua University Press and Springer-Verlag 2009. This article is published with open access at Springerlink.com
}

\begin{abstract}
Many potential applications of carbon nanotubes are expected to benefit from the availability of single-walled carbon nanotube materials enriched in metallic species, and specifically armchair nanotubes. The present work focuses on the modification of the pulsed laser vaporization (PLV) technique to selectively produce certain carbon nanotube structures. Nanotube nuclei were "warmed-up" with an additional laser pulse, timed to coincide approximately with the nucleation event. The effect of the second laser on the carbon vapor temperature was studied by emission spectroscopy. Nanotube type populations with and without warm-up were compared by means of absorption, photoluminescence, and Raman spectroscopy. It was found that the warm-up of nanotube nuclei with a laser pulse has a noticeable, albeit small, effect on the nanotube population. The intensity of spectral features associated with $(9,7)$ nanotube and its large chiral angle neighbors increased, while small chiral angle nanotubes decreased, with exception of the $(15,0)$ tube. This experiment demonstrates that nanotube population during PLV synthesis can be manipulated in a controlled fashion.
\end{abstract}

\section{KEYWORDS}

Nanotube synthesis, pulsed laser vaporization, nanotube population.

As with other nanomaterials, the electronic properties of single-walled carbon nanotubes (SWCNTs) strongly depend on their size and molecular structure, which can be conveniently expressed in terms of chiral indices $(n, m)$ [1]. Nanotubes with $n=m$ (armchair nanotubes) are metallic at room temperature while those with $n-m=3 j,(j=1,2$, $3 . .$.$) are strictly semi-metals, but often called metals$ along with armchairs. Nanotubes with $n-m=3 j+1$ and $n-m=3 j+2(j=0,1,2,3 \ldots)$ are semiconductors with a band gap that varies approximately inversely with diameter. Metallic and semi-metallic nanotubes exhibit singularities in the electron density of states similar to semiconducting ones, with corresponding optical transition energies inversely proportional to the diameter.

Such wealth of available electronic properties presents many opportunities to utilize SWCNTs

Address correspondence to pasha.nikolaev@gmail.com 
in various applications, such as field emission sources, transistors, interconnects on microchips, conductive transparent films, and photovoltaics [27]. Most of these applications will benefit from use of certain $(n, m)$ nanotube types (metallic, small gap semiconductor, etc.) However, as-produced SWCNT samples are polydisperse, with many $(n, m)$ types present and typical metal/semiconductor ratios of $\sim 1: 2$. This lack of specificity in SWCNT samples is one of the primary reasons why they are rarely used in commercial applications today. An ability to "tweak the knobs" on the SWCNT synthesis set-up to produce a desired narrow range of $(n, m)$ structures would greatly simplify the costly type separation [8], or even render it unnecessary. However, it seems that the only "knob" that has been touched so far is the synthesis temperature. The best known example is low temperature CoMoCat SWCNTs [9], that are naturally enriched in $(6,5),(7,5)$, and $(7,6)$ types. The SWCNTs produced by the alcohol chemical vapor deposition (CVD) technique [10] at lowered temperature are similarly enriched in the $(6,5),(7,5)$, and $(7,6)$ types. The SWCNT samples enriched in $(7,6)$ type were produced by the pulsed laser vaporization (PLV) technique on a $\mathrm{Rh} / \mathrm{Pd}$ catalyst [11] at a reduced temperature. Our recent results show that PLV production with a $\mathrm{Co} / \mathrm{Ni}$ catalyst at $900{ }^{\circ} \mathrm{C}$ results in enrichment in $(8,7),(9,7),(9,8)$ semiconducting and $(8,8),(9,9),(10,5)$ metallic nanotubes [12]. In all these cases the increased selectivity comes at lower than normal synthesis temperature, and at the expense of production yield.

Interestingly, all aforementioned selective production techniques make SWCNTs with chiral indices close to armchair structures. This suggests that the ultimate form of selectivity may be the production of pure armchair nanotubes. In fact, it has been reported that the CoMoCat technique produces significant quantities of $(6,6)$ and $(7,7)$ armchair structures in addition to predominantly $(6,5)$ [9]. The samples described in Refs. [10, 11] have not been analyzed for the presence of metallic nanotubes.

The PLV process for nanotube production [13, 14] was developed in the 1995 time frame and quickly became the technique of choice for production of high quality SWCNTs. The original claim of predominantly armchair metallic nanotubes present in these early samples [13] was based on electron beam diffraction (EBD) data [15]. Some EBD patterns were interpreted as SWCNT bundles consisting exclusively of armchair nanotubes. Nanotube EBD measurements have made a big leap forward since then [16], and these early pictures can now be interpreted as bundles with a range of chiral angles close to armchair structures. This generally agrees with recent EBD measurements on PLV nanotubes that show broad chiral angle distributions skewed towards armchair structures [17].

The explanation for this preference towards armchair structures is based on the energetics of SWCNT nucleation in the laser oven, the so-called "scooter" mechanism [13]. In the PLV process, a target made of the carbon/catalyst mixture is ablated with a high powered pulsed laser beam that instantly evaporates it into a plume of atomic/ molecular vapor. The major components of this plume are $\mathrm{C}_{2}, \mathrm{C}_{3}$ clusters, and atomic $\mathrm{Ni}$ and $\mathrm{Co}$. As the plume expands and cools down, the condensing carbon forms small planar sheets that curve into hemispherical clusters to minimize extra energy of the open carbon bonds along the sheet edge. In the absence of catalyst, most of these carbon clusters would close into fullerenes. But when catalyst atoms are present, one or several of them can land on the open edge of a carbon cluster, lowering its energy, and preventing it from closing for some time, thus allowing a nanotube to nucleate. As the nanotube nucleus grows to several hundred carbons and cools down rapidly, it loses fluidity and its structure becomes kinetically fixed. The nucleus diameter is largely determined by the energy balance between the open carbon edge and curvature-induced strain while the nucleus is still fluid [13, 18].

It has been shown $[13,19]$ that there are finer differences in the energy balance of a nanotube nucleus due to the energy difference between armchair and zig-zag edges. The zig-zag edges always have unsaturated bonds separated by two interatomic distances. On the armchair edge, however, unsaturated bonds are separated by only one interatomic distance, and if the structure is allowed to relax, they will form partial triple 
bonds, lowering the edge energy by approximately $0.8 \mathrm{eV}$ per double-coordinated carbon atom [19]. This means that a nanotube nucleus with a purely armchair edge is the most thermodynamically stable structure, the zig-zag edge is the least stable, and intermediate structures fall in between, with the edge energy difference approximately proportional to $(n-m)$. However, nanotube nuclei never reach any meaningful thermodynamic equilibrium, since nucleation is fast and driven by kinetics of the condensing plume. This results in nucleation of nanotubes with a broad range of chiral angles skewed towards thermodynamically preferential armchair structures instead of exclusively armchair types.

This reasoning suggests that changing the temperature history of the nanotube nuclei will have a profound effect on the nanotube type distribution. Expansion into colder buffer gas described in Ref. [11, 12] is the simplest case, as this simply increases the cooling rate. Assuming that nucleation occurs in the same temperature range, it has to happen faster in this case, leading to less contribution from kinetic factors that broaden type distribution, and hence to narrower type distributions [11, 12]. The "warm-up" approach explored in the present work is to add energy to nanotube nuclei after nuclei are initially formed, but before they are kinetically fixed, to allow them some extra time to remain fluid and move closer to thermodynamic equilibrium. The $1000{ }^{\circ} \mathrm{C}$ production temperature was chosen in order to reduce nanotube diameters, so that they are easily assessable by emission spectroscopy, and to give a narrower initial type distribution as well.

\section{Experimental}

Single-wall carbon nanotubes were produced in the PLV setup at NASA Johnson Space Center (JSC) as described elsewhere [20-23] under the following conditions: $\mathrm{Co} / \mathrm{Ni}$ catalyst ( 1 at $\%$ each), argon buffer gas at 500 Torr pressure, a flow rate of 100 sccm (standard cubic centimeters per minute) and temperature of $1000{ }^{\circ} \mathrm{C}$. Ablation was by the second harmonic $(532 \mathrm{~nm})$ of a Nd:YAG laser with $\sim 10 \mathrm{~ns}$ pulse width, $1.6 \mathrm{~J} / \mathrm{cm}^{2}$ energy density, and $60 \mathrm{~Hz}$ repetition rate. The first sample (no warm-up) was produced with the ablation laser only. A warm-up laser was added during production of the second sample: the third harmonic ( $355 \mathrm{~nm}$ ) of the Nd:YAG laser with $\sim 10 \mathrm{~ns}$ pulse width, $0.09 \mathrm{~J} / \mathrm{cm}^{2}$ energy density, $60 \mathrm{~Hz}$ repetition rate, delayed $500 \mu$ s after the ablation pulse. The warm-up beam was expanded to a diameter of $12 \mathrm{~mm}$ (compared to the $4 \mathrm{~mm}$ diameter of the ablation beam) to ensure that expanding vapor plume was entirely illuminated.

Black body temperature of the vapor plume was measured by means of emission spectroscopy. The 1 " flow tube of the PLV setup had two 3/4" side arms attached at $90^{\circ}$ angle ("X-tube" configuration). Side arms were sealed with optical quality quartz windows outside the oven. Emission spectra were acquired through one arm of the X-tube by projecting the plume image onto a $1 \mathrm{~mm}$ diameter optical fiber through a $60 \mathrm{~mm}$ focal length lens with 1:3 demagnification factor. This way, emission from a $\sim 3 \mathrm{~mm}$ region of the plume (collection region) was transferred to an SPEX 270 spectrometer (300 lines/mm grating centered at $510 \mathrm{~nm}, 25 \mu \mathrm{m}$ slit). Spectra were recorded with a Princeton Instruments ICCD detector with $10 \mu$ s exposure and averaged over 100 accumulations.

The samples collected from the PLV setup were dispersed in $\mathrm{D}_{2} \mathrm{O} / 1.5 \%$ sodium dodecylbenzenesulfonate (SDBS) surfactant by ultrasonication at $\sim 1 \mathrm{mg} / \mathrm{mL}$ concentration. The dispersions were centrifuged at 74,000 $g$ for $2 \mathrm{~h}$. The top $2 / 3^{\text {rd }}$ of the resulting supernatant was separated and used for photoluminescence and absorption spectroscopy measurements. Absorption spectra were acquired on Perkin Elmer Lambda 900 spectrometer in the 325-1600 nm wavelength range. Photoluminescence (PL) maps were acquired on a J-Y Spex Fluorolog 3-211 spectrometer equipped with an $\mathrm{LN}_{2}$-cooled InGaAs NIR detector. Excitation wavelength was varied in the $700-1000 \mathrm{~nm}$ range in 5 nm steps, and emission spectra were collected in the $1100-1150 \mathrm{~nm}$ range in $3 \mathrm{~nm}$ steps, with $5 \mathrm{~nm}$ slits. Raman spectra were acquired with 514 and $633 \mathrm{~nm}$ excitations on dry nanotubes using a Renishaw inVia spectrometer with a long working distance objective lens $(20 \times)$. 


\section{Results and discussion}

The $500 \mu$ s time delay between ablation and warmup laser pulses was selected in order to add energy to nanotube nuclei after nucleation, but before they are kinetically fixed. The accurate timing of this event is not known, and is impossible to assess by spectroscopy. A time delay of $500 \mu \mathrm{s}$ is the best guess based on the current understanding of the expansion dynamics of the laser plume, and the earlier diagnostics done at NASA JSC [24] and at Oak Ridge National Laboratory [25].

The plume is known to rapidly expand and travel away from the target following the ablation pulse. Therefore, plume position with respect to the target surface for various time delays was mapped in the following way: for each time delay the target position with respect to the fixed observation region was varied in 1-2 mm steps, and emission spectra were acquired. The plume positions were determined as maxima in the emission intensity, and were found to be 5,8 , and $11 \mathrm{~mm}$ for $0.1,0.5$, and 1 ms delays. respectively. Plume positions were interpolated for other time delays. In later spectral measurements, the target position was adjusted for each time delay so that the observation region coincided with the most luminous part of the plume

The plume emission up to $200 \mu$ s after the ablation pulse is dominated by the Swan band spectrum coming from excited $C_{2}{ }^{*}$ clusters and a small contribution from $\mathrm{C}_{3}{ }^{*}$ clusters (Fig. $1)$, in agreement with the literature [24]. At time delays longer than $300 \mu \mathrm{s}$, black body emission was detected. The energy density of the warm-up laser is smaller than that of the ablation laser by a factor of 18 , therefore no ablation of the target by the warm-up UV laser was expected, and none was detected. However, the possibility that the warm-up laser can evaporate carbon clusters and nanotube nuclei present in the plume (secondary ablation) could not be excluded. Therefore, emission spectra were acquired at a $500 \mu \mathrm{s}$ time delay with the warm-up laser on. Since no characteristic $C_{2}{ }^{*}$ emission was detected, it is evident that the secondary ablation threshold is above 0.09 $\mathrm{J} / \mathrm{cm}^{2}$.

Black body emission spectra for $0.5 \mathrm{~ms}$ green-UV delay were recorded at 300-1000 $\mu$ s time delays in 100 $\mu$ steps in order to track the temporal evolution of the plume temperature. Examples of emission spectra at $500 \mu$ s delay with and without the warm-up laser are shown in Fig. 2. The Boltzmann fits are shown as solid lines. The accuracy of Boltzmann fits is reduced by the uneven temperature distribution within the plume, and was estimated to be around $\pm 100-200$ $\mathrm{K}$, with lower accuracy at shorter time delays when larger temperature gradients are present. The time dependences of the black body temperature with and without the warm-up laser, calculated in this fashion, are shown in Fig. 3. The warm-up laser (its timing marked with an arrow) is producing an increase in the plume black body temperature of around $170 \mathrm{~K}$ for at least $100 \mu \mathrm{s}$. Unfortunately, we do not have any meaningful control over these parameters. A higher temperature increase will require a more powerful warm-up laser, with added risk of secondary ablation destroying nanotube nuclei. A longer temperature increase will probably require a warm-up source with pulse length greatly exceeding the $10 \mathrm{~ns}$ of a $\mathrm{Nd}$ : YAG laser, or multiple short-pulse sources. However, spectral measurements of the plume are necessary to establish that the warm-up laser does not destroy

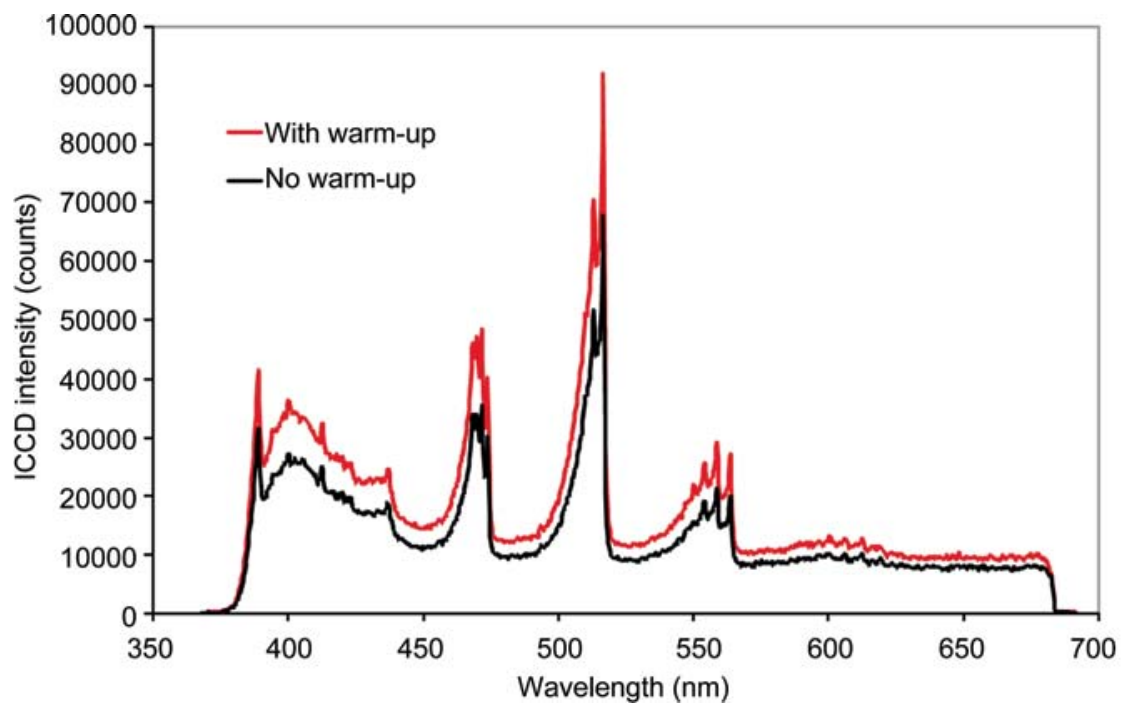

Figure 1 Emission spectra of the carbon vapor $100 \mu$ s after ablation, with (red) and without (black) warm-up pulse 


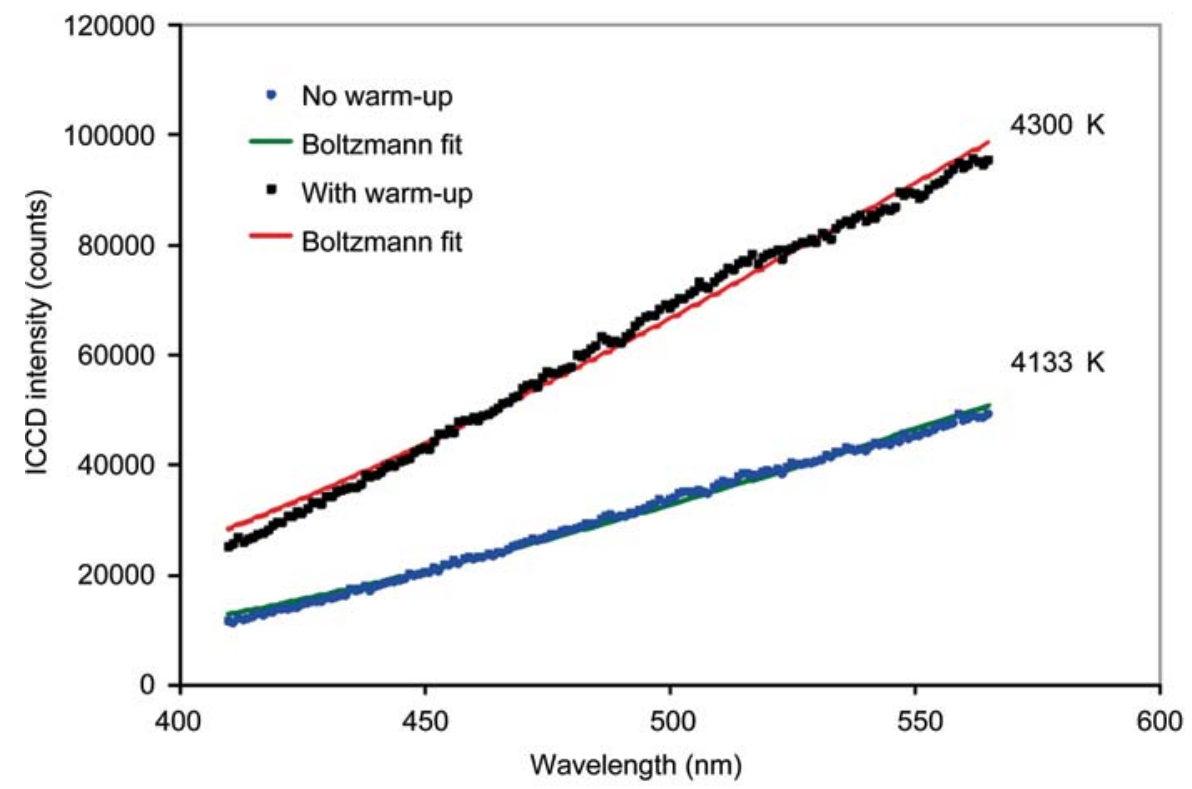

Figure 2 Emission spectra of the carbon vapor $500 \mu$ s after ablation, with (black) and without (blue) warm-up pulse. The corresponding Boltzmann fits are shown in red and green, respectively

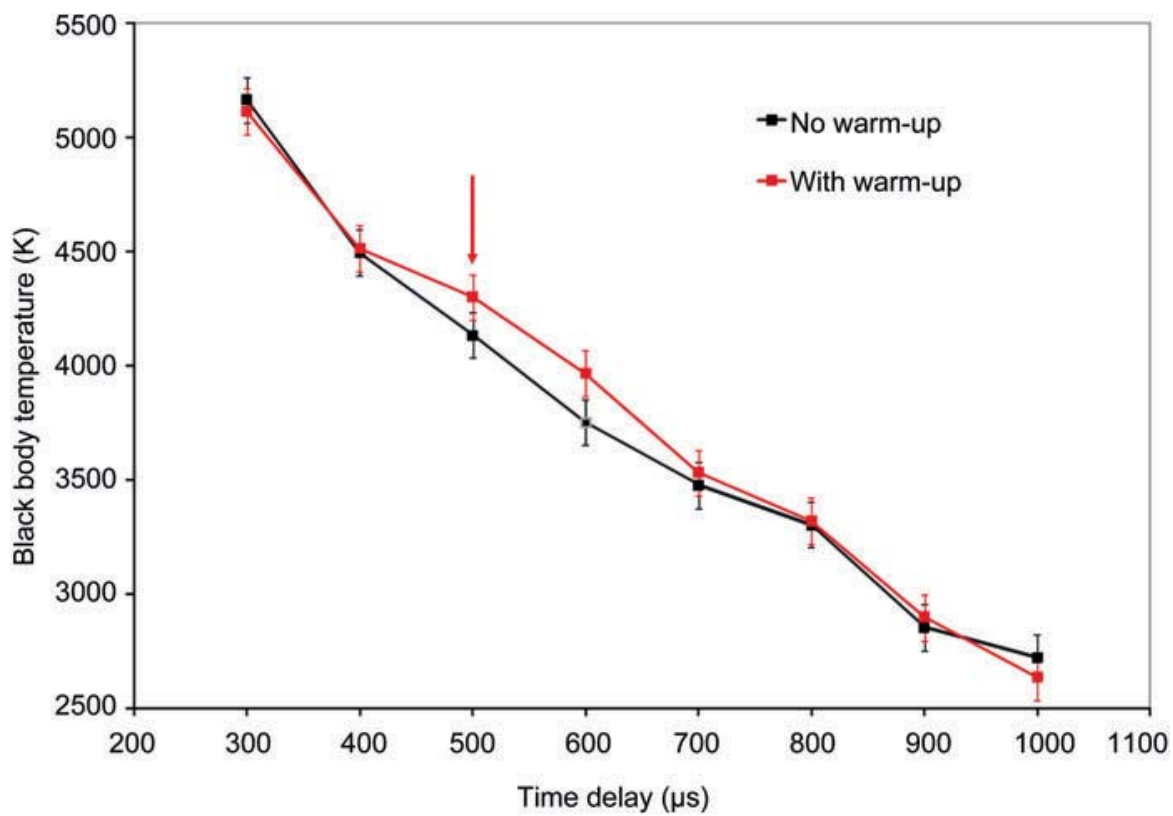

Figure 3 Black body temperature of the carbon vapor plume with (red) and without (black) warmup. The timing of the warm-up laser pulse is marked with an arrow

nanotube nuclei, and provides at least a moderate heating of the carbon vapor.

After the energy density and time delay of the warm-up laser were selected, two SWCNT samples were produced, without (1) and with (2) the warmup laser.

The PL maps of 1 and 2 are shown in Fig. 4, and appear very similar. A total of 14 distinct peaks corresponding to 14 semiconducting nanotube structures were detected. In order to observe small differences in peak intensity, each sample was dispersed in surfactant solution and measured twice, and each map was fit with 14 two-dimensional Lorentzian line shapes. No background subtraction was necessary. Averages and standard deviations of normalized amplitudes were calculated and compared. The PL intensities are plotted in Fig. 5 with respect to diameter and chiral angle. There is no 


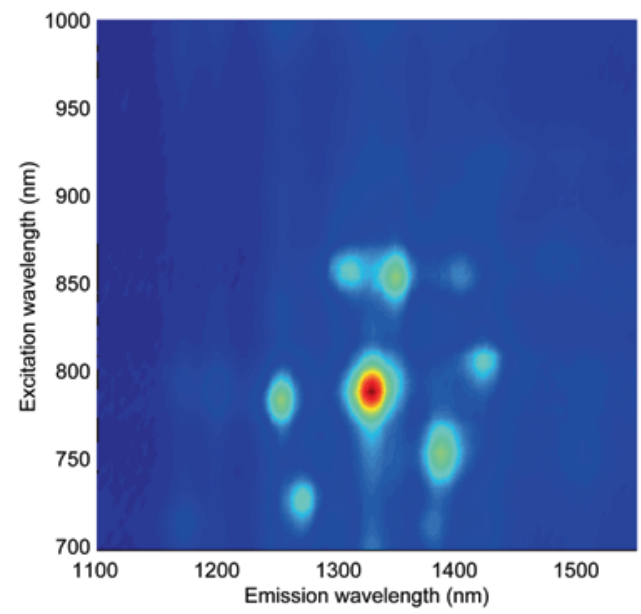

(a)

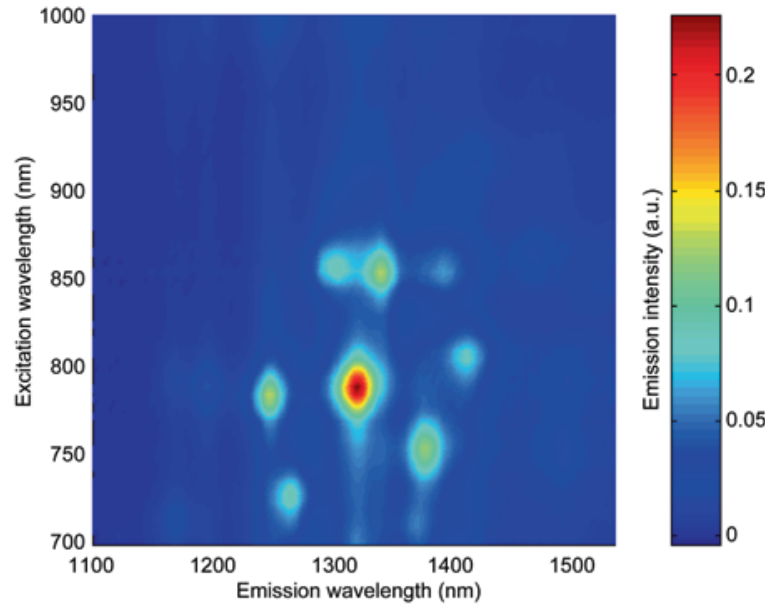

(b)

Figure 4 Photoluminescence maps of nanotube samples synthesized without (a) and with (b) warm-up

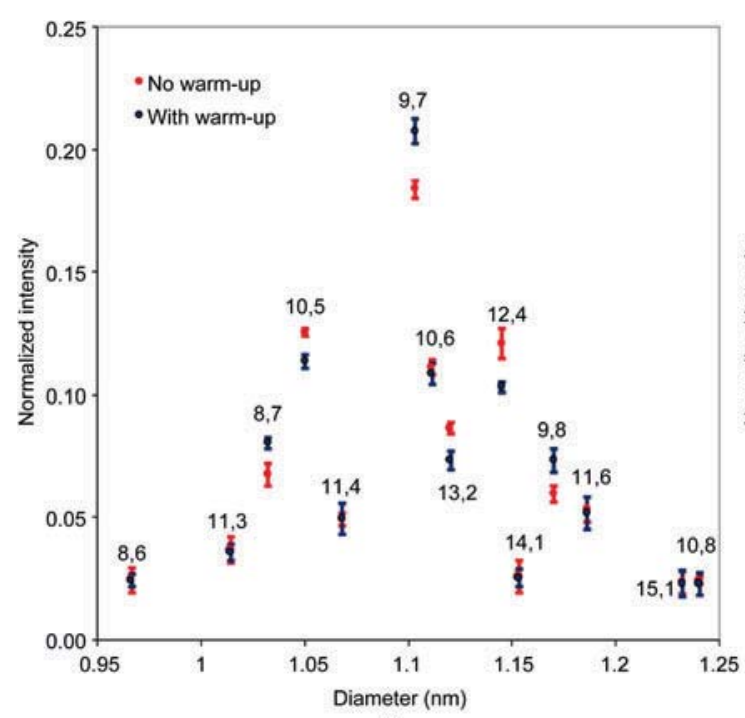

(a)

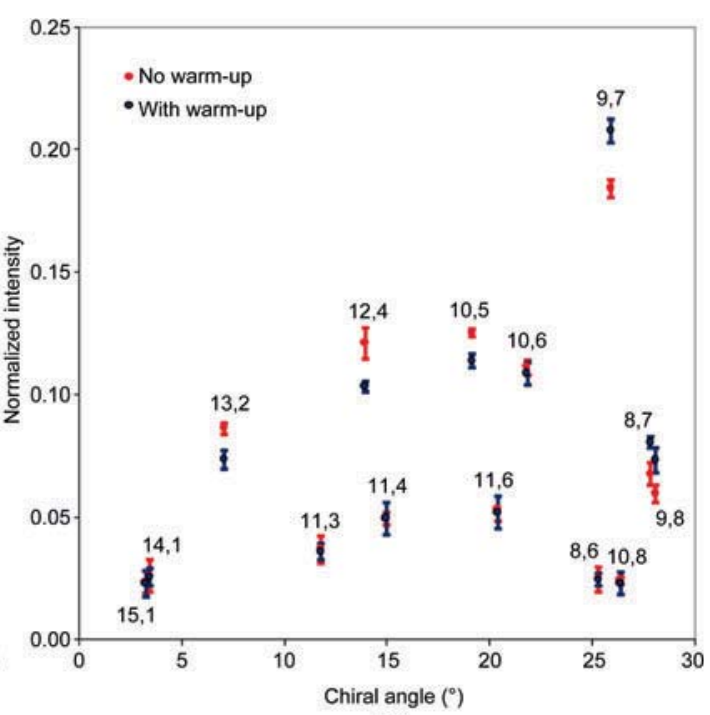

(b)

Figure 5 Normalized PL intensities of semiconducting nanotubes with respect to nanotube diameter (a) and chiral angle (b)

clear diameter dependence of the intensity change; however, there is a clear chiral angle dependence: nanotubes with the closest to armchair chiral angles $\left(>25^{\circ}\right)$, in this case $(9,7),(9,8)$, and $(8,7)$, have increased PL intensity in the sample synthesized with the warm-up laser. Nanotubes with smallest chiral angles $\left(<20^{\circ}\right)$, such as $(13,2),(12,4)$, and $(10,5)$, have decreased PL intensity in this sample. Relative differences in PL intensity are rather small, in the $9 \%-23 \%$ range, but clearly exceed the standard deviations.

Absorption spectra of $\mathbf{1}$ and $\mathbf{2}$ with linear background subtracted are shown in Fig. 6. The $\mathrm{S}_{22}$ transition wavelengths of the 14 identified semiconducting nanotubes are marked in black, with arrows indicating increase/decrease in the PL intensity of 2 with respect to $\mathbf{1}$. Changes in the absorption of the spectral features at 805 and 865 $\mathrm{nm}$ are in agreement with the PL intensity changes of the constituent nanotubes. Note that there are at least 40 semiconducting nanotubes with $S_{22}$ transition energies that could be constituents of the absorption spectra of $\mathbf{1}$ and $\mathbf{2}$, and without PL maps it would be impossible to discriminate their presence in the sample by deconvolution of the spectra into Lorentzian line shapes.

The 16 metallic nanotubes that might be present in $\mathbf{1}$ and $\mathbf{2}$ are marked above the $\mathrm{M}_{11}$ region of the absorption spectra in Fig. 6. Their absorption features group into three distinct peaks, each consisting 
of five or six nanotube types. Since metallic nanotubes do not photoluminesce, metallic tubes were discriminated using Raman spectroscopy. The available Raman excitation lines, 514 and $633 \mathrm{~nm}$, are marked in Fig. 6. It is evident that the $633 \mathrm{~nm}$ line will be in resonance exclusively with a number of metallic tubes, while the $514 \mathrm{~nm}$ line will be in resonance with fewer metallic nanotubes, and with few largest diameter semiconducting tubes on $S_{33}$ transitions as well. Examples of radial breathing mode (RBM) regions of Raman spectra of $\mathbf{1}$ and $\mathbf{2}$ with 633 and 514 excitations are shown in Fig. 7, and demonstrate small differences in the relative peak intensities. Four spectra for each excitation were acquired for each sample and deconvoluted into a number of mixed Lorentzian-Gaussian line shapes after flat background subtraction based on the flat spectrum in the $300-700 \mathrm{~cm}^{-1}$ spectral range. RBM frequencies are known to shift by a few wavenumbers due to bundling and environmental effects [27]; therefore, peak positions and widths were determined for one spectrum, and for the subsequent spectra peak positions were allowed to float within $1.5 \mathrm{~cm}^{-1}$, while their widths were held constant. After

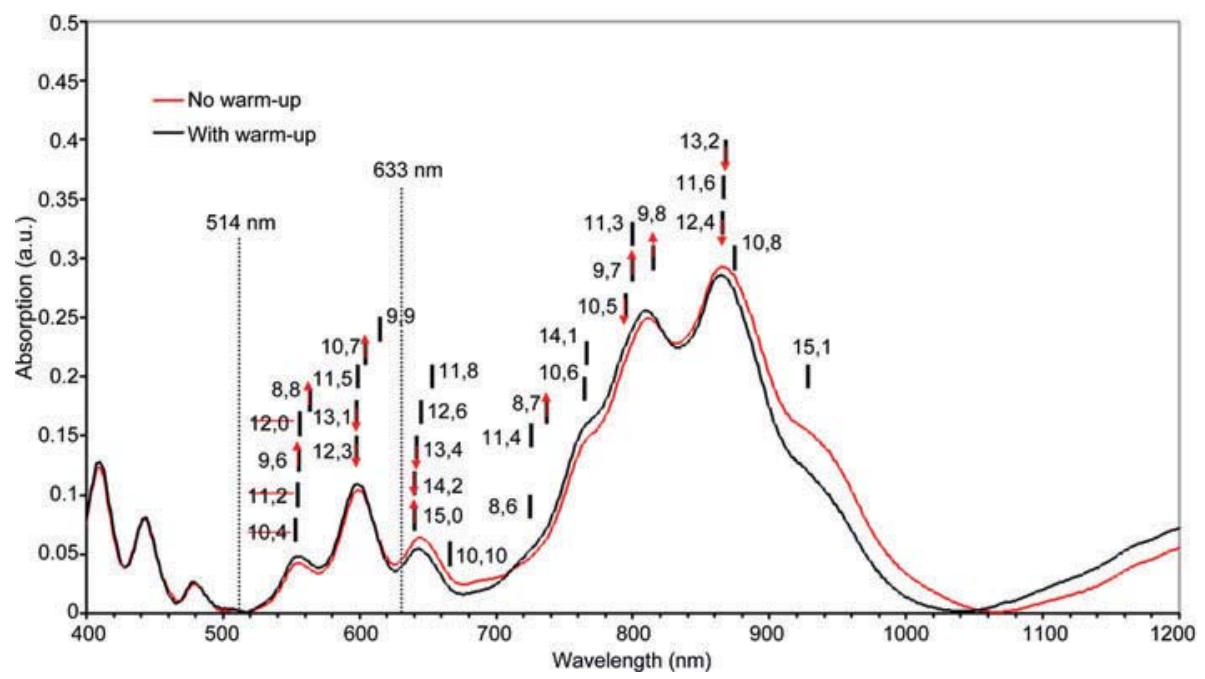

Figure 6 Absorption spectra of nanotube samples synthesized with and without warm-up. The arrows indicate increase / decrease in the PL intensity of the sample peaks after warm-up

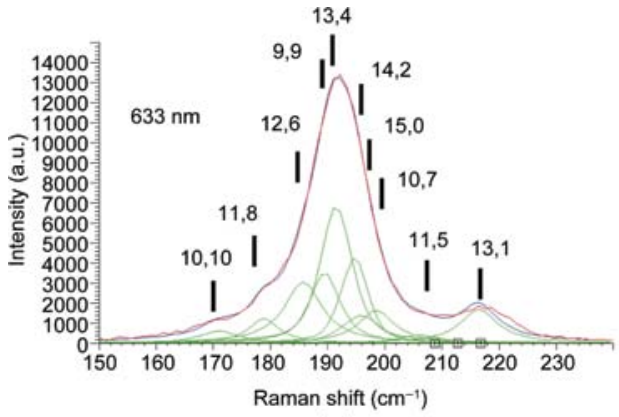

(a)

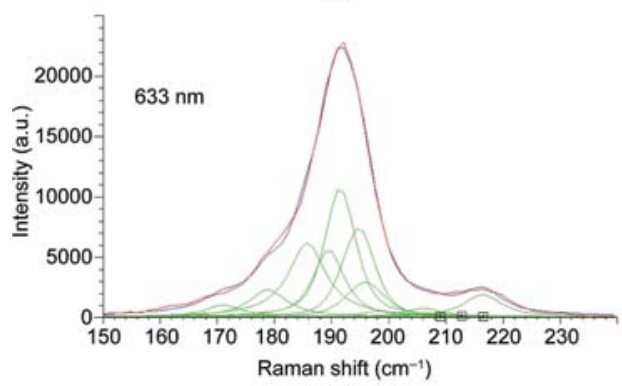

(c)

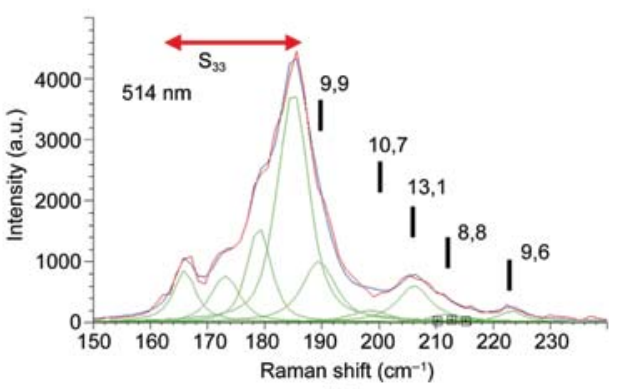

(b)

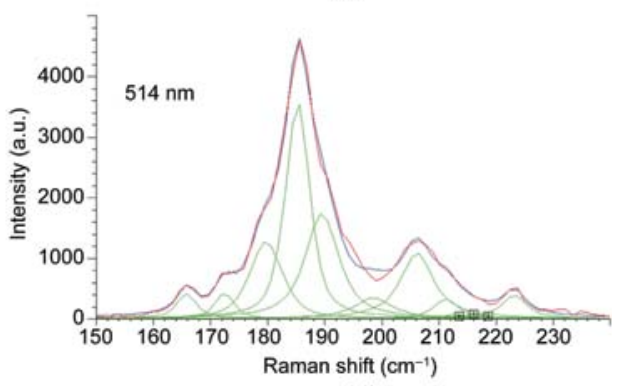

(d)

Figure 7 Raman spectra of nanotube samples synthesized without $(a, b)$ and with $(c, d)$ warm-up 
deconvolution, peak areas were normalized and average intensities and their standard deviations were calculated. Peaks were associated with their respective $(n, m)$ types by identifying an "anchor" tube on each spectrum $((13,1)$ for $633 \mathrm{~nm}$ excitation; $(9,6)$ and $(13,1)$ for $514 \mathrm{~nm}$ excitation), and mapping the rest of them under the assumption that RBM frequency is inversely proportional to nanotube diameter. It was found that RBM frequencies are downshifted by $3-5 \mathrm{~cm}^{-1}$ compared to ones experimentally determined by tunable Raman spectroscopy [26] on free-standing SWCNT carpets. The RBM intensities were further adjusted to account for nanotubes being excited off resonance. The PL excitation profiles of semiconducting nanotubes are known [28]. It was assumed that Raman excitation profiles are similar in shape, and that widths and positions of the main line and overtones scale proportionally to the transition energy. Using this way, an estimate of the excitation profile was calculated for each metallic nanotube, and its RBM intensity was adjusted according to the position of the Raman laser line on the profile.

The adjusted RBM intensities for $\mathbf{1}$ and $\mathbf{2}$ are plotted with respect to diameter and chiral angle in Fig. 8. There is no clear diameter dependence of the intensity change; however, there is a clear chiral angle dependence: nanotubes with the closest to armchair chiral angles $\left(>23^{\circ}\right)$, in this case $(8,8),(10,7)$, and $(9,6)$, have increased RBM intensity in the sample synthesized with the warm-up laser. Nanotubes with

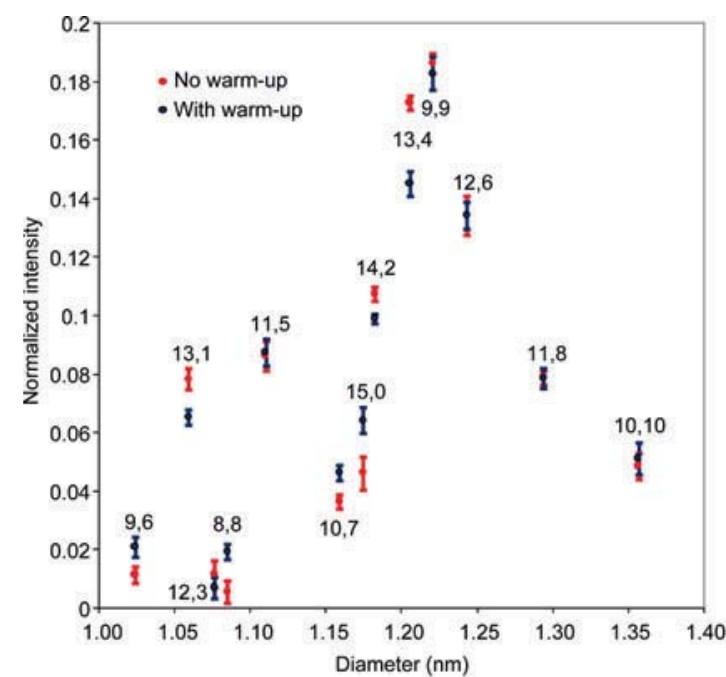

(a) smallest chiral angles $\left(<15^{\circ}\right)$, such as $(13,4),(14,2)$, and $(13,1)$, have decreased RBM intensity in this sample. A notable exception from this trend is the zig -zag $(15,0)$ nanotube; its RBM intensity is higher in 2. Relative differences in RBM intensities are $20 \%$ $80 \%$ range, reaching $240 \%$ for the $(8,8)$ nanotube, and clearly exceed the standard deviations.

The chiral map of $\mathbf{1}$ is shown in Fig. 9. Semiconducting nanotubes are marked with green circles, with area proportional to PL intensity, while metallic tubes are marked with red circles with area proportional to adjusted Raman intensity. The relative contributions of metallic and semiconducting nanotubes are normalized under the common assumption that $1 / 3^{\text {rd }}$ of them are metallic. The increases and decreases in nanotube intensities in $\mathbf{2}$ are marked with up and down arrows. Overall, the most intense $(9,7)$ nanotube and five of its neighbors increase as a result of warm-up of the nanotube nuclei, along with the $(15,0)$ metallic tube. Seven nanotubes with chiral angles in the $3^{\circ}-19^{\circ}$ range decrease in intensity. It is important to emphasize that this chiral map reliably shows only changes in the intensities of the spectral features of the corresponding nanotubes. The absolute concentrations of nanotubes are proportional to their intensities, but the relationships between nanotube concentration and intensity of its spectral features are not accurately known, and are known to vary quite significantly among nanotube types [29-32]. In addition, semiconducting and

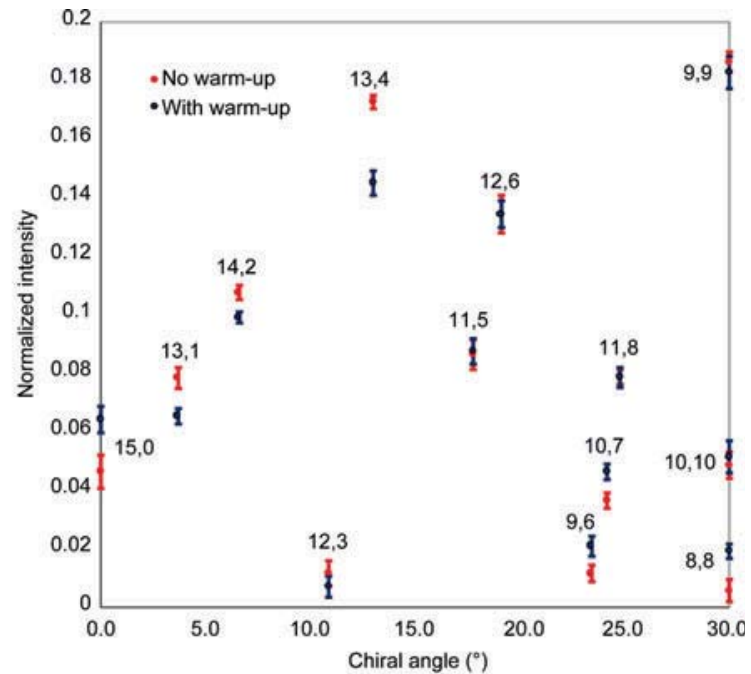

(b)

Figure 8 Normalized Raman intensities of metallic nanotubes with respect to nanotube diameter (a) and chiral angle (b) 


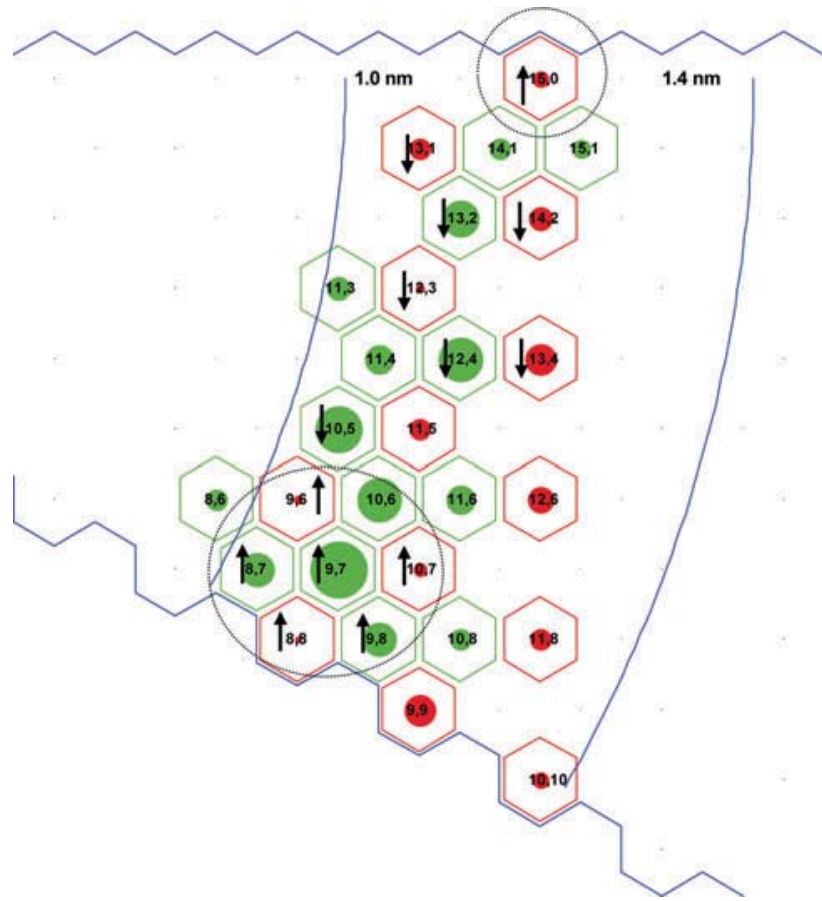

Figure 9 Population map comparison. Semiconducting nanotubes are marked with green circles, with area proportional to PL intensity, while metallic tubes are marked with red circles with area proportional to adjusted Raman intensity. The increases and decreases in nanotube intensities after warm-up are marked with up and down arrows

metallic nanotubes are mapped by different types of spectroscopy, PL, and Raman.

\section{Conclusions}

Warm-up of nanotube nuclei with a pulsed laser in a PLV production setup has a noticeable, albeit small, effect on the nanotube population. Increase in the spectral intensities of nanotube species with armchair and close-to-armchair chiral angles supports the idea that adding energy to nanotube nuclei after initial formation, but before they are kinetically fixed, allows them some extra time to remain fluid and move closer to the thermodynamically preferential armchair structure. The magnitude of the warmup effect on the nanotube population is small for several reasons. First, the amplitude and duration of the temperature increase that could be achieved with the pulsed Nd:YAG laser is rather modest. Second, since nanotube nucleation occurs in a certain temperature window, and temperature distribution within a plume is not homogenous, some nuclei may be receiving the warm-up energy either too early or too late to be affected. And the third, most important, reason is that the choice of the time delay (500 $\mu \mathrm{s})$ is rather arbitrary, and probably far from optimal. However, this experiment demonstrates that nanotube population during PLV synthesis can be manipulated in a controlled fashion. Additional experiments using long pulse lasers will be necessary to achieve better control.

\section{Acknowledgements}

The authors would like to acknowledge financial support from NASA under contract \#NNJ05HI05C and from State of Texas, Space Act Agreement \#SAAAT-07-021 (RAN 0798) (UTA07-099). The authors thank Dr. R. Bruce Weisman and Saunab Ghosh of Rice University for help with photoluminescence measurements.

\section{References}

[1] Dresselhaus, M. S.; Dresselhaus, G.; Avouris, P. Carbon Nanotubes: Synthesis, Structure, Properties and Applications. Topics in Applied Physics; Springer-Verlag: Heidelberg, 2001; Vol. 80.

[2] Anantram, M. P.; Leonard, F. Physics of carbon nanotube electronic devices. Rep. Prog. Phys. 2006, 69, 507-561.

[3] Avouris, P.; Chen, Z. H.; Perebeinos, V. Carbon-based electronics. Nat. Nanotechnol. 2007, 2, 605-615.

[4] Thostenson, E. T.; Ren, Z. F.; Chou, T. W. Advances in the science and technology of carbon nanotubes and their composites: A review. Compos. Sci. Technol. 2001, 61, 1899-1912.

[5] Kaushik, B. K.; Goel, S.; Rauthan, G. Future VLSI interconnects: Optical fiber or carbon nanotube-A review. Microelectron. Int. 2007, 24, 53-63.

[6] Mahar, B.; Laslau, C.; Yip, R.; Sun, Y. Development of carbon nanotube-based sensors - A review. IEEE Sens. J. 2007, 7, 266-284.

[7] Charlier, J. C.; Blase, X.; Roche, S. Electronic and transport properties of nanotubes. Rev. Mod. Phys. 2007, 79, 677-732.

[8] Hersam, M. C. Progress towards monodisperse singlewalled carbon nanotubes. Nat. Nanotechnol. 2008, 3, 387-394.

[9] Lolli, G.; Zhang, L.; Balzano, L.; Sakulchaicharoen, 
N.; Tan, Y.; Resasco, D. E. Tailoring $(n, m)$ structure of single-walled carbon nanotubes by modifying reaction conditions and the nature of the support of CoMo catalysts. J. Phys. Chem. B 2006, 110, 2108-2115.

[10] Miyauchi, Y.; Chiashi, S.; Murakami, Y.; Hayashida, Y.; Maruyama, S. Fluorescence spectroscopy of single-walled carbon nanotubes synthesized from alcohol. Chem. Phys. Lett. 2004, 387, 198-203.

[11] Suzuki, S.; Asai, N.; Kataura, H.; Achiba, Y. Formation of single-wall carbon nanotubes in $\mathrm{Ar}$ and nitrogen gas atmosphere by using laser furnace technique. Eur. Phys. J.

D 2007, 43, 143-146.

[12] Nikolaev, P.; Holmes, W.; Sosa, E.; Boul, P.; Arepalli, S.; Yowell, L. Effect of vaporization temperature on the diameter and chiral angle distributions of single-wall carbon nanotubes. J. Nanosci. Nanotech., in press.

[13] Thess, A.; Lee, R.; Nikolaev, P.; Dai, H. J.; Petit, P.; Robert, J.; Xu, C. H.; Lee, Y. H.; Kim, S. G.; Rinzler, A. G. et. al. Crystalline ropes of metallic carbon nanotubes. Science 1996, 273, 483-487.

[14] Guo, T.; Nikolaev, P.; Thess, A.; Colbert, D. T.; Smalley, R. E. Catalytic growth of single-walled nanotubes by laser vaporization. Chem. Phys. Lett. 1995, 243, 49-54.

[15] Cowley, J. M.; Nikolaev, P.; Thess, A.; Smalley, R. E. Electron nano-diffraction study of carbon single-walled nanotube ropes. Chem. Phys. Lett. 1997, 265, 379-384.

[16] Jiang, H.; Nasibulin, A. G.; Brown, D. P.; Kauppinen, E. I. Unambiguous atomic structural determination of singlewalled carbon nanotubes by electron diffraction. Carbon 2007, 45, 662-667.

[17] Jiang, H.; Brown, D. P.; Nikolaev, P.; Nasibulin, A. G.; Kauppinen, E. I. Determination of helicities in unidirectional assemblies of graphitic or graphitic-like tubular structures. Appl. Phys. Lett. 2008, 93, 141903.

[18] Nikolaev, P. Gas Phase Production of Single Wall Carbon Nanotubes. Ph. D. thesis, Rice University, Houston, TX, 2000.

[19] Lee, Y. H.; Kim, S. G.; Tománek, D. Catalytic growth of single-wall carbon nanotubes: An ab initio study. Phys. Rev. Lett. 1997, 78, 2393-2396.

[20] Arepalli, S.; Holmes, W. A.; Nikolaev, P.; Hadjiev, V. G.; Scott, C. D. A parametric study of single-wall carbon nanotube growth by laser ablation. J. Nanosci. Nanotech. 2004, 4, 762-773.

[21] Arepalli, S.; Scott, C. Spectral measurements in production of single-wall nanotubes by laser ablation.
Chem. Phys. Lett. 1999, 302, 139-145.

[22] Arepalli, S.; Nikolaev, P.; Holmes, W.; Scott, C. D. Diagnostics of laser produced plume under carbon nanotube growth conditions. Appl. Phys. A 2000, 70, 125-133.

[23] Arepalli, S. Laser ablation process for single wall carbon nanotube production. J. Nanosci. Nanotech. 2004, 4, 317-325.

[24] Scott, C. D.; Arepalli, S.; Nikolaev, P.; Smalley, R. E. Growth mechanisms for single-wall carbon nanotubes in a laserablation process. Appl. Phys. A 2001, 72, 573-580.

[25] Puretzky, A. A.; Schittenhelm, H.; Fan, Xu.; Lance, M. J; Allard, L. F. Jr.; Geohegan, D. B. Investigations of singlewall carbon nanotube growth by time-restricted laser vaporization. Phys. Rev. B 2002, 65, 245425.

[26] Araujo, P. T.; Doorn, S. K.; Kilina, S.; Tretiak, S.; Einarsson, E.; Maruyama, S.; Chacham, H.; Pimenta, M. A.; Jorio, A. Third and fourth optical transitions in semiconducting carbon nanotubes. Phys. Rev. Lett. 2007, 98, 067401.

[27] Fantini, C.; Jorio, A.; Souza, M.; Strano, M. S.; Dresselhaus, M. S.; Pimenta, M. A. Optical transition energies for carbon nanotubes from resonant Raman spectroscopy: Environment and temperature effects. Phys. Rev. Lett. 2004, 93, 147406.

[28] Weisman, R. B.; Bachilo, S. M. Dependence of optical transition energies on structure for single-walled carbon nanotubes in aqueous suspension: An empirical Kataura plot. Nano Lett. 2003, 3, 1235-1238.

[29] Jiang, J.; Saito, R.; Gruneis, A.; Dresselhaus, G.; Dresselhaus, M. S. Optical absorption matrix elements in single-wall carbon nanotubes. Carbon 2004, 42, 3169 3176.

[30] Oyama, Y.; Saito, R.; Sato, K.; Jiang, J.; Samsonidze, Ge. G.; Gruneis, A.; Miyauchi, Y.; Maruyama, S.; Jorio, A.; Dresselhaus, G.; Dresselhaus, M. S. Photoluminescence intensity of single-wall carbon nanotubes. Carbon 2006, 44, 873-879.

[31] Jiang, J.; Saito, R.; Sato, K.; Park, J. S.; Samsonidze, Ge. G.; Jorio, A.; Dresselhaus, G.; Dresselhaus, M. S. Excitonphoton, exciton-phonon matrix elements, and resonant Raman intensity of single-wall carbon nanotubes. Phys. Rev. B 2007, 75, 035405.

[32] Tsyboulski, D. A.; Rocha, J. -D. R.; Bachilo, S. M.; Cognet, L.; Weisman, R. B. Structure-dependent fluorescence efficiencies of individual single-walled carbon nanotubes. Nano Lett. 2007, 7, 3080-3085. 